\title{
Exploring the Influencing Factors for Infant Mortality: A Mixed-Method Study of 24 Developing Countries Based on Demographic and Health Survey data
}

\author{
Md. Akhtarul Islam ${ }^{1}$, Tarana Tabassum ${ }^{1}$, and Mohammad Ali Moni² \\ ${ }^{1}$ Khulna University \\ ${ }^{2}$ The University of Sydney
}

February 15, 2021

\begin{abstract}
Objective: This study aimed to discover the prevalence of infant mortality and to assess how different factors influence infant mortality in 24 developing countries by utilizing the latest DHS data. Methods: This study used a mixed-method design to assemble cross-sectional studies to integrate data from 24 other countries due to a widening perspective of infant mortality. Most recent available DHS data of 24 different developing countries from the year 2013 to 2019 was used to conduct the study. Descriptive analysis, binary logistic regression model, random-effect meta-analysis, and forest plot have been used for the final analyses. Results: Binary logistic regression model revealed for Bangladesh that, higher education level of fathers (OR: 0.344, 95\% CI: 0.147 ; 0.807), being 2nd born or above order infant (OR: $0.362,95 \%$ CI: $0.248,0.527$ ), taking ANC (OR: $0.271,95 \%$ CI: $0.192 ; 0.382$ for $1-4$ visits), taking PNC (OR: $0.303,95 \%$ CI: $0.216 ; 0.425)$ were statistically significant determinants of lowering infant death. While carrying multiple fetus (OR: 6.634, 95\% CI: 3.247; 13.555) was exposed as a risk factor of infant mortality. Most significant factors influencing infant mortality for all 24 developing countries were number of fetus (OR: 0.193, 95\% CI: $0.176 ; 0.213$ ), taking ANC (OR: 0.356, 95\% CI: $0.311 ; 0.407)$ and taking PNC (OR: $0.302,95 \%$ CI: $0.243 ; 0.375)$. Conclusion In this study, the number of the fetus, taking ANC and PNC, was the most significant factor affecting the risk of infant mortality in developing countries. So, anticipation and control projects ought to be taken in the field in regard to these hazard factors.
\end{abstract}

\section{Introduction}

The infant mortality rate (IMR), characterized as the number of deaths in youngsters under one year of age for every 1000 live births, has been viewed as a profoundly delicate measure of public health ${ }^{1}$. Infant mortality is found to be one of the most significant parts of under-five child mortality as a vulnerable age group for medicinal administrations, which is particularly necessary for the foundation of wellbeing, social prosperity, and endorsement of standard life ${ }^{2-7}$. The mortality rate of infants underneath one year is one of the most deciding indicators of a nation's advancement ${ }^{8}$. In 1990, 9 million children younger than one year died globally. Till 2011 every year, about 4,000,000 infants used to die during the initial month of life, and worldwide neonatal mortality made up $40 \%$ of the complete child mortality ${ }^{9}$. About $99 \%$ of these deaths occurred in developing countries, particularly in sub-Saharan Africa and South Asia ${ }^{10-12}$. Consequently, decreasing youngster mortality along with the advancement of healthy lives and prosperity for all children has been one of the principles worldwide difficulties in the course of the most recent years, and decreasing it by $66 \%$ between 1990 and 2015 has become the fourth Millennium Development Goal (MDG) of the United Nations (UN). Similarly, under sustainable improvement objectives (SDG), the countries expect to diminish preventable deaths of infants to necessarily as low as 12 for every 1,000 live births and under-five youngsters mortality to as low as 25 for every 1,000 live births ${ }^{13,14}$. As a result of the United Nations Millennium 
Development Goals (MDGs), where reduction of the infant mortality rate was a key challenge, the rate of infant mortality reduced from 65 fatalities for every 1000 live births to 29 deaths for every 1000 by $2015^{15-18}$. Nevertheless, an estimate explicated that 6.3 million children died in 2017, in most cases from preventable causes. About 1.6 million of these deaths occurred at age 1-11 months, with 2.5 million deaths happening in the very first month of life. African countries are holding higher IMR rather than developed countries like European countries. Fifty-three infant deaths per 1000 live births in sub-Saharan Africa whereas three infant deaths per 1000 live births in the European Union in 2018 ${ }^{17-19}$.

Bangladesh is a small south Asian country that is still underdeveloped. Bangladesh has gained tremendous betterment towards youngster wellbeing in the previous decade ${ }^{20}$. In spite of this decrease in newborn child mortality, the level of infant death rate isn't optimal in Bangladesh contrasted with the other developing countries $^{21}$. The infant mortality rate was 38 for every 1000 live births in the year 2014 though it was 43 and 52 in 2011 and 2007 respectively in Bangladesh ${ }^{22}$.

Besides, in developing countries, socio-economic condition and health-related factors such as place of residence, education level of parents, wealth index, maternal age at delivery, birth order number, child's size, antenatal care utilization, birth weight, type of infant nutrition, the status of breastfeeding, delivery assistance and many more factors are found to be responsible for the likelihood of infant death ${ }^{8,23-29}$.

In this paper, considering the vital facts related to infant mortality, we tried to apprehend the influencing factors of infant mortality in 24 developing countries, including Bangladesh. We introduced a mixed method that was designated to combine data from Bangladesh and 23 other countries, which we think would be more effective to give more insights about infant death than using a simple cross-sectional survey and metaanalysis.

\section{Methods}

\subsection{Design}

We applied a mixed-method design to conduct the study. A binary logistic regression (BLR) was conducted for the cross-sectional study of Bangladesh. Afterward, we made a comparison between the results from Bangladesh and the findings of a meta-analysis of 24 developing countries. We could broadly explore the influential determinants of infant mortality by employing this approach. All the data were taken from the Demographic and Health Survey (DHS).

\subsection{Data source and data extraction}

Demographic and Health Survey (DHS) data is collected using a cross-sectional study design for a large nationally representative sample for every country. Similar questionnaires and the same measures are used to collect the information from the respondent. To select study respondents in most of these surveys, a two-stage cluster sampling design with households in urban and rural strata has been utilized. Detailed information about the sampling and data collection methodology is available on the DHS websites ${ }^{30}$. Initially, for this cross-sectional study, we extracted relevant information for analysis from a nationwide representative secondary dataset, Bangladesh Demography and Health Survey 2014, for binary logistic regression ${ }^{21}$. Besides, we conducted a meta-analysis utilizing the recently accessible datasets (accessed in January 2020) from MEASURE DHS. We adopted the recent available DHS data for the 24 developing countries ${ }^{16}$. Which is Afghanistan (2015), Angola (2015-16), Benin (2017-18), Chad (2014-15), Cambodia (2014), Egypt (2014), Ethiopia (2016), Guinea (2018), India (2015-16), Indonesia (2017), Kenya (2014), Lesotho (2014), Malawi (2015-16), Myanmar (2015-16), Nepal (2016), Nigeria (2018), Pakistan (2017-18), Sierra Leone (2013), South Africa (2016), Tanzania Timor-Leste (2016), Zambia (2013-14), Zimbabwe (2015). The DHS database contains information from 91 countries (http:// dhsprogram.com/data/available-datasets.com); 67 countries were excluded due to excessive missing values and unavailability of information regarding dependent and independent variables in any of the selected countries. Finally, we decided on 24 developing countries that are homogeneous.

\subsection{Variables}


In this study, we considered infant mortality as the dependent variable. We measured this as a two-category dummy variable, and the two distinct levels are "yes " if infant death occurs and "no " if death doesn't occur. We included a group of relevant socio-economic and demographic factors as an independent variable to execute the research and to discover the impacting determinants that are presumed to influence infant mortality based on the previous literature. Commencing with the type of place of residence, which has remained the same as the existing category of DHS datasets. Similarly, the category ofmaternal current working status remained the same as the original datasets. The remaining covariates were subcategorized. We mergedno education and primary to up to primary for BDHS data, whereas the other categories were secondary andhigher in terms of parent's education level. Again, for meta-analysis, we combined secondary and higher toabove primary while another category was up to the primary. In the case of binary logistic regression, the wealth index has remained the same as the original data. Further, we changed the label of the variable to Living below the poverty line for the meta-analysis with two categories. We combined poorer andpoorest and labeled them as 'yes', which means if the individuals are poor, they certainly live below the poverty line, on the other hand, we combined middle, richer, and richest with the label 'no ', which represents the individuals who live above the poverty line. The variable birth order number was categorized asfirstborn and $2^{\text {nd }}$ and above born for both binary logistic regression and meta-analysis. Maternal age at delivery was converted into a nominal scale from the continuous form with the category less than or equal to 19 years (<=19)and above 19 years ${ }^{30}$. Categories for the variable number of fetus were single and multiplefor both methods. For binary logistic regression, we subcategorizedtaking antenatal care (ANC) as no visits , 1-4 visits, more than 4 visits, whereas in the meta-analysis, individuals who had at least one $A N C$ visit were considered in the category yes, otherwise no. We used the original variabletaking postnatal care $(P N C)$ that was categorized as two levels,yes and no, for both logistic regression and meta-analysis. Finally, for the child's size, we subcategorized the variable as average, larger than average (combined by very large and larger than average), and smaller than average (combined by very small and smaller than average) for the logistic regression analysis. We further recoded this variable for meta-analysis with two independent levels, such as average and larger or smaller than average .

\subsection{Statistical analysis}

We used statistical software SPSS V.23 (SPSS Inc. Chicago, USA) and R V.3.6.2 (Bell Laboratories, New Jersey, USA) to carry out the analysis. Binary logistic regression was practiced to determine the key factors that have an impact on infant mortality in Bangladesh using BDHS data ${ }^{31,32}$. Besides, we applied metaanalysis on the DHS data from Bangladesh and 23 other developing countries ${ }^{33}$. Heterogeneity was assessed by enumerating values from $\mathrm{I}^{2}$ and $\mathrm{p}$ values among datasets ${ }^{34,35}$. We performed a random-effects model in the meta-analytical approach as significant heterogeneity was found by which we estimated DerSimonian and Laird's pooled effect ${ }^{36}$. Forest plots were used to display $95 \%$ CI, summary measure, and weight of each study for the most significant determinants ${ }^{37}$. As a summary measure, we used Odds Ratio (OR), and all findings were weighted to handle bias due to under-sampling and oversampling ${ }^{38}$.

\section{Results}

Table 1 shows the baseline characteristics of the selected covariates for BDHS data. Most of the respondents are from a rural area (about $68.2 \%$ ). Up to primary education is available for $41.5 \%$ women and $53.5 \%$ men and only $11.5 \%$ of the women and $15.2 \%$ of the men have higher education. Also, we find about $78.1 \%$ of women are not working currently, and $75.3 \%$ of women are above 19 years old at delivery time. Most of the respondents (98.7\%) included in the analysis carry a single fetus. Again $67.4 \%$ of children are average in size, and $19.6 \%$ are smaller than average, while $61.6 \%$ are taken for postnatal check-ups. About $54.5 \%$ of respondents visit 1-4 times for antenatal care, and $25.5 \%$ of respondents do not go for the check-ups.

Table 1 Baseline characteristics of different variables with levels for BDHS data 


\begin{tabular}{|c|c|c|c|}
\hline Variable & $\begin{array}{l}\text { Levels of the variables } \\
\text { with code }\end{array}$ & Frequency & Percentage \\
\hline $\begin{array}{l}\text { Dependent variable } \\
\text { Infant mortality }\end{array}$ & No [0] Yes [1] & 4561163 & 96.53 .5 \\
\hline $\begin{array}{l}\text { Covariates Type of } \\
\text { place of residence }\end{array}$ & Rural [0] Urban [1] & 32201504 & 68.231 .8 \\
\hline Maternal education & $\begin{array}{l}\text { Up to primary }[0] \\
\text { Secondary }[1] \text { Higher }[2]\end{array}$ & 19592224541 & 41.547 .011 .5 \\
\hline Father's education & $\begin{array}{l}\text { Up to primary }[0] \\
\text { Secondary [1] Higher }[2]\end{array}$ & 25261478720 & 53.531 .315 .2 \\
\hline Wealth index & $\begin{array}{l}\text { Poorest [0] Poorer [1] } \\
\text { Middle [2] Richer [3] } \\
\text { Richest [4] }\end{array}$ & 1011902901980930 & 21.419 .119 .120 .719 .7 \\
\hline $\begin{array}{l}\text { Maternal current working } \\
\text { status }\end{array}$ & No $[0]$ Yes $[1]$ & 36891035 & 78.121 .9 \\
\hline Birth order number & $\begin{array}{l}\text { First born [0] Second and } \\
\text { above [1] }\end{array}$ & 19432781 & 41.158 .9 \\
\hline Maternal age at delivery & $<=19[0]$ Above $19[1]$ & 11683556 & 24.775 .3 \\
\hline Number of fetus & Single [0] Multiple [1] & 466262 & 98.71 .3 \\
\hline Taking ANC & $\begin{array}{l}\text { No [0] } 1-4 \text { visits [1] More } \\
\text { than } 4 \text { visits [2] }\end{array}$ & 12042573947 & 25.554 .520 .0 \\
\hline Taking PNC & No $[0]$ Yes $[1]$ & 18162908 & 38.461 .6 \\
\hline Size of child & $\begin{array}{l}\text { Average [0] Larger than } \\
\text { average [1] Smaller than } \\
\text { average [2] }\end{array}$ & 3181616927 & 67.413 .019 .6 \\
\hline
\end{tabular}

The baseline characteristics of the selected factors for 24 developing countries are displayed in table 2 . We calculated the prevalence of all 24 countries separately with the DHS's sampling weights. Table 3 demonstrates the different influential socio-economic and demographic determinants of infant mortality in Bangladesh. Infant mortality is associated with maternal education with an adjusted OR of 0.537 (95\% CI 0.380 to $0.759: \mathrm{p} \leq 0.001)$ for the secondary level of education. Individuals from poorer and middle-class households show a significant influence on infant mortality, where the ORs are 0.448 (95\% CI 0.303 to 0.663 ; $\mathrm{p} \leq 0.001)$ and $0.408(95 \%$ CI 0.254 to $0.654 ; \mathrm{p} \leq 0.001)$ for poorer and middle-class household, respectively. Similarly, birth order number has a noticeable impact on infant mortality with an adjusted OR of 0.362 $(95 \%$ CI 0.248 to $0.527: \mathrm{p} \leq 0.001)$ with respect to $2^{\text {nd }}$ and above born infants. Mothers aged above 19 years significantly influence infant mortality with an adjusted OR 0.477 (95\% CI 0.333 to $0.682: \mathrm{p} \leq 0.001)$. The variable number of fetus shows a significant association with infant mortality, where the OR for the category' multiple fetus' is 6.634 (95\% CI 3.247 to 13.555: $\mathrm{p} \leq 0.001)$. Likewise, taking ANC has a significant influence on infant mortality, with an OR of 0.271 (95\% CI 0.192 to $0.382: \mathrm{p} \leq 0.001)$ for the category' $1-4$ visits'. The OR of taking PNC is 0.303 (95\% CI 0.216 to 0.425 ; $\leq \leq 0.001$ ), which exhibits an association between infant mortality and taking PNC services. The sizes of children have a significant impact on infant mortality with an adjusted OR 0.578 (95\% CI 0.400 to $0.834 ; \mathrm{p}=0.003$ ) regarding the category 'smaller than average'.

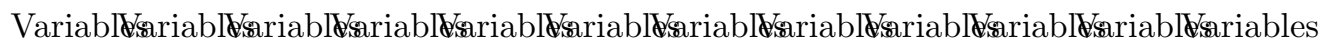




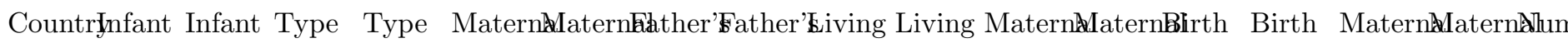
Name mor- mor- of of Ed- Ed- Ed- Ed- be- be- Cur- Cur- Or- Or- Age Age of

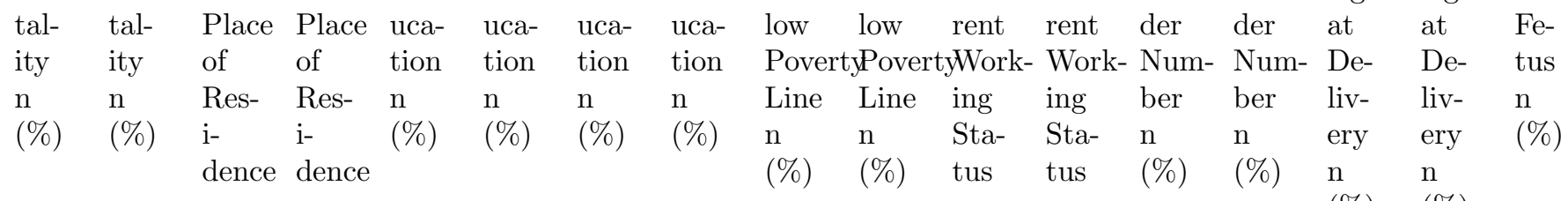
$\begin{array}{lllllll}\mathrm{n} & \mathrm{n} & \mathrm{n} & \mathrm{n} & (\%) & (\%)\end{array}$

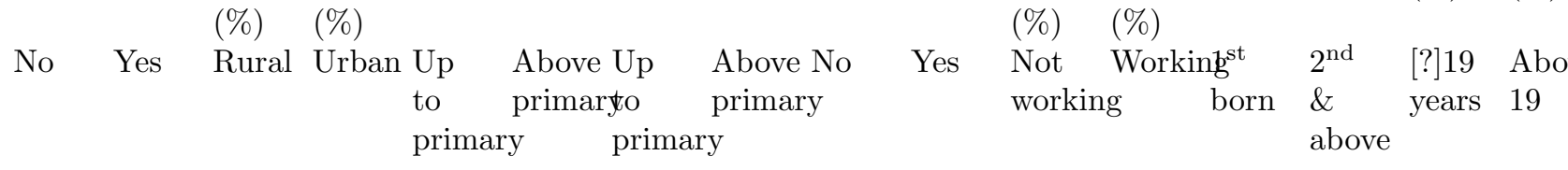

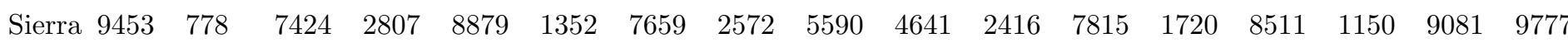

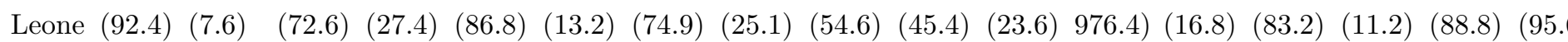
2013

$\begin{array}{llllllllllllllllll}\text { Zambial1164 } & 469 & 7425 & 4208 & 8061 & 3572 & 5748 & 5885 & 5997 & 5636 & 4761 & 6872 & 1880 & 9753 & 1233 & 10400 & 1123\end{array}$

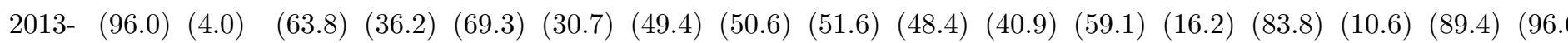
14

$\begin{array}{lllllllllllllllll}\text { Banglad6́61 } & 163 & 3220 & 1504 & 1959 & 2765 & 2526 & 2198 & 2811 & 1913 & 3689 & 1035 & 1943 & 2781 & 1168 & 3556 & 4662\end{array}$

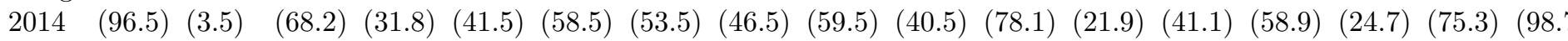
$\begin{array}{lllllllllllllllll}\text { Cambo69.3 } & 164 & 5148 & 1929 & 4504 & 2573 & 3732 & 3345 & 4072 & 3005 & 2360 & 4717 & 2744 & 4333 & 491 & 6586 & 6945\end{array}$

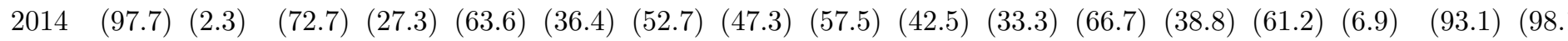

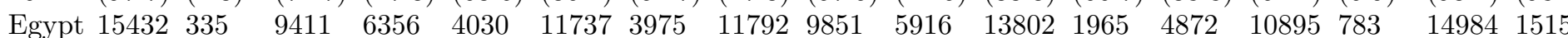
$\begin{array}{lllllllllllllllllll} & 014 & (97.9) & (2.1) & (59.7) & (40.3) & (25.6) & (74.4) & (25.2) & (74.8) & (62.5) & (37.5) & (87.5) & (12.5) & (30.9) & (69.1) & (5.0) & (95.0) & (96 .\end{array}$

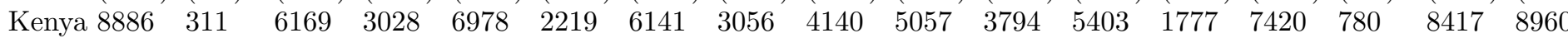

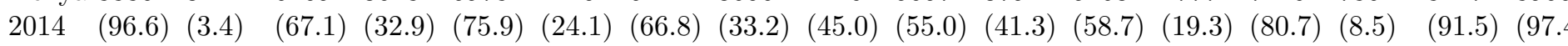

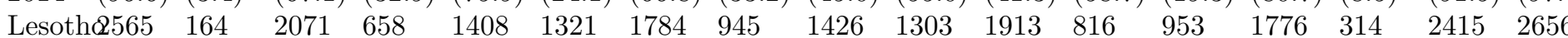

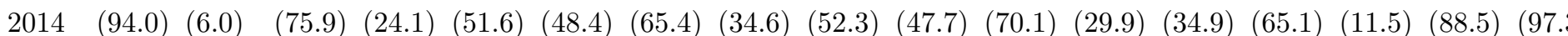

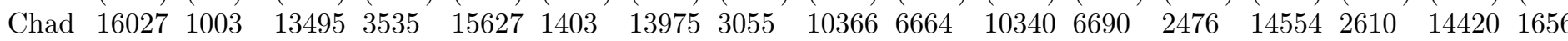

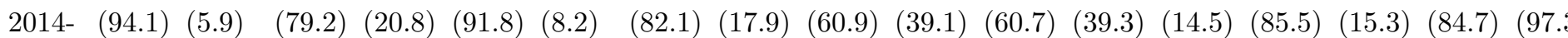
15

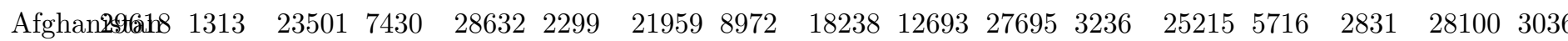

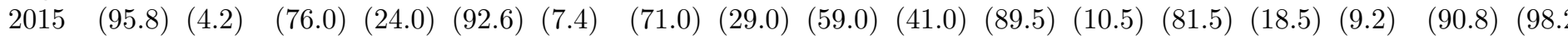

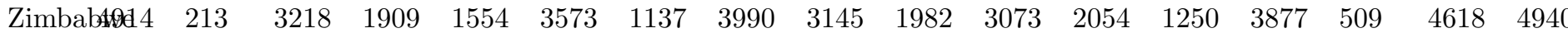

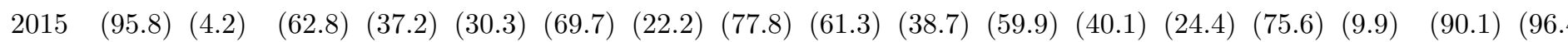

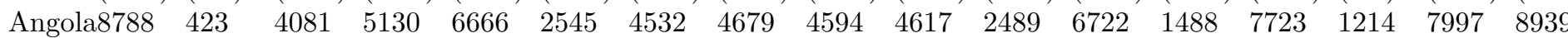

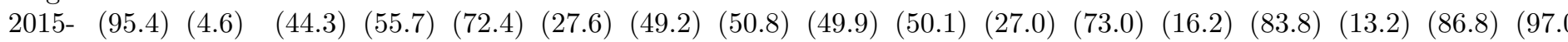
16

$\begin{array}{lllllllllllllllll}\text { Malawi13603 } & 522 & 11892 & 2233 & 11176 & 2949 & 9132 & 4993 & 8115 & 6010 & 4878 & 9247 & 3330 & 10795 & 1891 & 12234 & 1359\end{array}$ 2015- (96.3) (3.7) (84.2) (15.8) (79.1) (20.9) (64.7) (35.3) (57.5) (42.5) (34.5) (65.5) (23.6) (76.4) (13.4) (86.6) (96. 16

$\begin{array}{lllllllllllllllll}\text { Tanzan8a91 } & 319 & 6668 & 1842 & 7033 & 1477 & 6798 & 1712 & 4707 & 3803 & 1962 & 6548 & 1733 & 6777 & 843 & 7667 & 8211\end{array}$

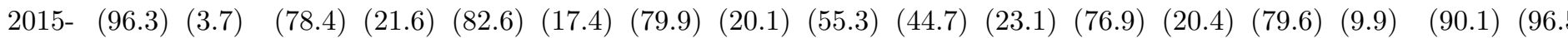
16

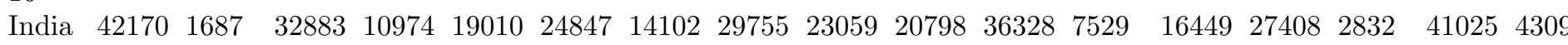

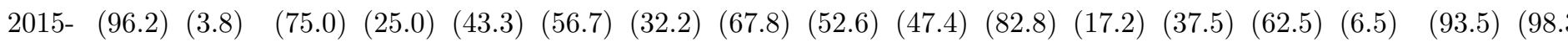
16 


\begin{tabular}{|c|c|c|c|c|c|c|c|c|c|c|c|c|c|c|c|}
\hline $\begin{array}{l}\text { 29Myaн4135. } \\
2015-\quad(95.9) \\
16\end{array}$ & $\begin{array}{l}187 \\
(4.1)\end{array}$ & $\begin{array}{l}3564 \\
(78.5)\end{array}$ & $\begin{array}{l}976 \\
(21.5)\end{array}$ & $\begin{array}{l}2791 \\
(61.5)\end{array}$ & $\begin{array}{l}1749 \\
(38.5)\end{array}$ & $\begin{array}{l}2603 \\
(57.3)\end{array}$ & $\begin{array}{l}1937 \\
(42.7)\end{array}$ & $\begin{array}{l}2156 \\
(47.5)\end{array}$ & $\begin{array}{l}2384 \\
(52.5)\end{array}$ & $\begin{array}{l}2133 \\
(47.0)\end{array}$ & $\begin{array}{l}2407 \\
(53.0)\end{array}$ & $\begin{array}{l}1475 \\
(32.5)\end{array}$ & $\begin{array}{l}3065 \\
(67.5)\end{array}$ & $\begin{array}{l}218 \\
(4.8)\end{array}$ & $\begin{array}{l}4322 \\
(95.2)\end{array}$ \\
\hline $\begin{array}{l}\text { South } 1351 \\
\text { Africa } \\
016\end{array}$ & $\begin{array}{l}46 \\
(3.3)\end{array}$ & $\begin{array}{l}556 \\
(39.8)\end{array}$ & $\begin{array}{l}841 \\
(60.2)\end{array}$ & $\begin{array}{l}178 \\
(12.7)\end{array}$ & $\begin{array}{l}1219 \\
(87.3)\end{array}$ & $\begin{array}{l}225 \\
(16.1)\end{array}$ & $\begin{array}{l}1172 \\
(83.9)\end{array}$ & $\begin{array}{l}783 \\
(56.0)\end{array}$ & $\begin{array}{l}614 \\
(44.0)\end{array}$ & $\begin{array}{l}913 \\
(65.4)\end{array}$ & $\begin{array}{l}484 \\
(34.6)\end{array}$ & $\begin{array}{l}297 \\
(21.3)\end{array}$ & $\begin{array}{l}1100 \\
(78.7)\end{array}$ & $\begin{array}{l}58 \\
(4.2)\end{array}$ & $\begin{array}{l}1339 \\
(95.8)\end{array}$ \\
\hline $\begin{array}{l}\text { thiopi } 9372 \\
016\end{array}$ & $\begin{array}{l}455 \\
(4.6)\end{array}$ & $\begin{array}{l}8099 \\
(82.4)\end{array}$ & $\begin{array}{l}1728 \\
(17.6)\end{array}$ & $\begin{array}{l}8811 \\
(89.7)\end{array}$ & $\begin{array}{l}1016 \\
(10.3)\end{array}$ & $\begin{array}{l}8053 \\
(81.9)\end{array}$ & $\begin{array}{l}1774 \\
(18.1)\end{array}$ & $\begin{array}{l}4464 \\
(45.4)\end{array}$ & $\begin{array}{l}5363 \\
(54.6)\end{array}$ & $\begin{array}{l}7246 \\
(73.7)\end{array}$ & $\begin{array}{l}2581 \\
(26.3)\end{array}$ & $\begin{array}{l}1874 \\
(19.1)\end{array}$ & $\begin{array}{l}7953 \\
(80.9)\end{array}$ & & $\begin{array}{l}8961 \\
(91.2)\end{array}$ \\
\hline $\begin{array}{ll}\text { Jepal } & 4821 \\
016 & (96.9)\end{array}$ & $\begin{array}{l}155 \\
(3.1)\end{array}$ & $\begin{array}{l}2145 \\
(43.1)\end{array}$ & $\begin{array}{l}2831 \\
(56.9)\end{array}$ & $\begin{array}{l}2642 \\
(53.1)\end{array}$ & $\begin{array}{l}2334 \\
(46.9)\end{array}$ & & $\begin{array}{l}3161 \\
(63.5)\end{array}$ & & $\begin{array}{l}2380 \\
(47.8)\end{array}$ & $\begin{array}{l}2399 \\
(48.2)\end{array}$ & $\begin{array}{l}2577 \\
(51.8)\end{array}$ & $\begin{array}{l}1958 \\
(39.3)\end{array}$ & & & $\begin{array}{l}4216 \\
(84.7)\end{array}$ \\
\hline Timor-5580 & 154 & 3801 & 1933 & 2229 & 3505 & 2290 & 3444 & 3697 & 2037 & 3604 & 2130 & 1453 & 4281 & 275 & 5459 \\
\hline $\begin{array}{ll}\text { Leste } & (97.3) \\
2016 & \end{array}$ & $(2.7)$ & $(66.3)$ & $(33.7)$ & $(38.9)$ & $(61.1)$ & $(39.9)$ & $(60.1)$ & $(64.5)$ & $(35.5)$ & $(62.9)$ & $(37.1)$ & $(25.3)$ & $(74.7)$ & $(4.8)$ & $(95.2)$ \\
\hline $\begin{array}{l}\text { ndonesi6487 } \\
017 \quad(97.9)\end{array}$ & $\begin{array}{l}347 \\
(2.1)\end{array}$ & $\begin{array}{l}8452 \\
(50.2)\end{array}$ & $\begin{array}{l}8382 \\
(49.8)\end{array}$ & $\begin{array}{l}4330 \\
(25.7)\end{array}$ & $\begin{array}{l}12504 \\
(74.3)\end{array}$ & $\begin{array}{l}4587 \\
(27.2)\end{array}$ & $\begin{array}{l}12247 \\
(72.8)\end{array}$ & $\begin{array}{l}9054 \\
(53.8)\end{array}$ & $\begin{array}{l}7780 \\
(46.2)\end{array}$ & $\begin{array}{l}9059 \\
(53.8)\end{array}$ & $\begin{array}{l}7775 \\
(46.2)\end{array}$ & $\begin{array}{l}5468 \\
(32.5)\end{array}$ & $\begin{array}{l}11366 \\
(67.5)\end{array}$ & & $\begin{array}{l}16031 \\
(95.2)\end{array}$ \\
\hline $\begin{array}{ll}\text { Senin } & 11379 \\
017- & (95.4)\end{array}$ & $\begin{array}{l}549 \\
(4.6)\end{array}$ & $\begin{array}{l}7326 \\
(61.4)\end{array}$ & $\begin{array}{l}4602 \\
(38.6)\end{array}$ & $\begin{array}{l}10066 \\
(84.4)\end{array}$ & $\begin{array}{l}1862 \\
(16.6)\end{array}$ & $\begin{array}{l}9110 \\
(76.4)\end{array}$ & $\begin{array}{l}2818 \\
(23.6)\end{array}$ & $\begin{array}{l}6716 \\
(56.3)\end{array}$ & $\begin{array}{l}5212 \\
(43.7)\end{array}$ & $\begin{array}{l}2292 \\
(19.2)\end{array}$ & $\begin{array}{l}9636 \\
(80.8)\end{array}$ & $\begin{array}{l}2387 \\
(20.0)\end{array}$ & $\begin{array}{l}9541 \\
(80.0)\end{array}$ & & $\begin{array}{l}10988 \\
(92.1)\end{array}$ \\
\hline $\begin{array}{l}\text { Pakistan1845 } \\
2017-\quad(94.9) \\
18\end{array}$ & $\begin{array}{l}638 \\
(5.1)\end{array}$ & $\begin{array}{l}6960 \\
(55.8)\end{array}$ & $\begin{array}{l}5523 \\
(44.2)\end{array}$ & $\begin{array}{l}8086 \\
(64.8)\end{array}$ & $\begin{array}{l}4397 \\
(35.2)\end{array}$ & $\begin{array}{l}5204 \\
(41.7)\end{array}$ & $\begin{array}{l}7279 \\
(58.3)\end{array}$ & $\begin{array}{l}6785 \\
(54.4)\end{array}$ & $\begin{array}{l}5698 \\
(45.6)\end{array}$ & $\begin{array}{l}11046 \\
(88.5)\end{array}$ & $\begin{array}{l}1437 \\
(11.5)\end{array}$ & $\begin{array}{l}2964 \\
(23.7)\end{array}$ & $\begin{array}{l}9519 \\
(76.3)\end{array}$ & $\begin{array}{l}786 \\
(6.3)\end{array}$ & $\begin{array}{l}11697 \\
(93.7)\end{array}$ \\
\hline $\begin{array}{l}\text { Guinea6854 } \\
2018 \quad(93.7)\end{array}$ & $\begin{array}{l}462 \\
(6.3)\end{array}$ & $\begin{array}{l}5374 \\
(73.5)\end{array}$ & $\begin{array}{l}1942 \\
(26.5)\end{array}$ & $\begin{array}{l}6518 \\
(89.1)\end{array}$ & $\begin{array}{l}798 \\
(10.9)\end{array}$ & $\begin{array}{l}5931 \\
(81.1)\end{array}$ & $\begin{array}{l}1385 \\
(18.9)\end{array}$ & $\begin{array}{l}3817 \\
(52.2)\end{array}$ & $\begin{array}{l}3499 \\
(47.8)\end{array}$ & $\begin{array}{l}2340 \\
(32.0)\end{array}$ & $\begin{array}{l}4976 \\
(68.0)\end{array}$ & $\begin{array}{l}1267 \\
(17.3)\end{array}$ & $\begin{array}{l}6049 \\
(82.7)\end{array}$ & $\begin{array}{l}995 \\
(13.6)\end{array}$ & $\begin{array}{l}6321 \\
(86.40\end{array}$ \\
\hline Nigeria29359 & 1950 & 20664 & 10645 & 19339 & 11970 & 15963 & 15346 & 16608 & 14701 & 10278 & 21031 & 5625 & 25684 & 2922 & 28387 \\
\hline $2018 \quad(93.8)$ & $(6.2)$ & $(66.0)$ & $(34.0)$ & $(61.8)$ & $(38.2)$ & $(51.0)$ & $(49.0)$ & $(53.0)$ & $(47.0)$ & $(32.8)$ & $(67.2)$ & $(18.0)$ & $(82.0)$ & $(9.3)$ & $(90.7)$ \\
\hline
\end{tabular}

Table 2 Baseline characteristics of selected covariates for 24 developing countries

Table 3 Results of the binary logistic regression model affecting socio-economic and demographic factors for infant mortality in Bangladesh.

\begin{tabular}{lllll}
\hline & $B^{*}$ & Odds Ratio & $p$-value & $95 \%$ CI for OR \\
\hline $\begin{array}{l}\text { Type of place of } \\
\text { residence Rural } \\
\text { Ref. Category) }\end{array}$ & -0.101 & 0.904 & 0.606 & {$[0.616 ; 1.326]$} \\
$\begin{array}{l}\text { Urban } \\
\text { Maternal }\end{array}$ & $-0.622-0.723$ & 0.5370 .485 & & \\
$\begin{array}{l}\text { Education Up to } \\
\text { Primary (Ref. }\end{array}$ & & 0.0020 .0000 .116 & {$[0.380 ; 0.759]$} \\
$\begin{array}{l}\text { Category) } \\
\text { Secondary Higher }\end{array}$ & & & & {$[0.197 ; 1.196]$} \\
$\begin{array}{l}\text { Father's } \\
\text { Education Up to }\end{array}$ & $-0.413-1.067$ & 0.6620 .344 & 0.0170 .0400 .014 & \\
$\begin{array}{l}\text { Primary (Ref. } \\
\text { Category) }\end{array}$ & & & & \\
$\begin{array}{l}\text { Secondary Higher } \\
\text { Wealth Index }\end{array}$ & $-0.802-0.897$ & 0.4480 .4080 .812 & 0.0000 .0000 .000 & {$[0.147 ; 0.980]$} \\
$\begin{array}{l}\text { Poorest (Ref. } \\
\text { Category) Poorer }\end{array}$ & $-0.209-0.049$ & 0.952 & 0.3890 .874 & {$[0.303 ; 0.663]$} \\
$\begin{array}{l}\text { Middle Richer } \\
\text { Richest }\end{array}$ & & & & \\
\end{tabular}




\begin{tabular}{|c|c|c|c|c|}
\hline & $B^{*}$ & Odds Ratio & $p$-value & $95 \%$ CI for OR \\
\hline $\begin{array}{l}\text { Maternal } \\
\text { Current Working } \\
\text { Status Not } \\
\text { Working (Ref. } \\
\text { Category) Working }\end{array}$ & 0.027 & 1.027 & 0.883 & {$[0.716 ; 1.475]$} \\
\hline $\begin{array}{l}\text { Birth Order } \\
\text { Number First } \\
\text { Born (Ref. } \\
\text { Category) Second } \\
\text { and above }\end{array}$ & -1.016 & 0.362 & 0.000 & {$[0.248,0.527]$} \\
\hline $\begin{array}{l}\text { Maternal Age at } \\
\text { Delivery } \\
<=19 \text { (Ref. } \\
\text { Category) Above } 19\end{array}$ & -0.741 & 0.477 & 0.000 & {$[0.333 ; 0.682]$} \\
\hline $\begin{array}{l}\text { Number of Fetus } \\
\text { Single (Ref. } \\
\text { Category) Multiple }\end{array}$ & 1.892 & 6.634 & 0.000 & {$[3.247 ; 13.555]$} \\
\hline $\begin{array}{l}\text { Taking } \\
\text { Antenatal Care } \\
\text { No (Ref. } \\
\text { Category) } 1-4 \\
\text { Visits More than } \\
4 \text { Visits }\end{array}$ & $-1.306-0.758$ & 0.2710 .468 & 0.0000 .0000 .003 & $\begin{array}{l}{[0.192 ; 0.382]} \\
{[0.286 ; 0.768]}\end{array}$ \\
\hline $\begin{array}{l}\text { Taking Postnatal } \\
\text { Care No (Ref. } \\
\text { Category) Yes }\end{array}$ & -1.194 & 0.303 & 0.000 & {$[0.216 ; 0.425]$} \\
\hline $\begin{array}{l}\text { Size of Child } \\
\text { Average (Ref. } \\
\text { Category) Larger } \\
\text { than Average } \\
\text { Smaller than } \\
\text { Average }\end{array}$ & $0.366-0.548$ & 1.4430 .578 & $\begin{array}{lll}0.001 & 0.078 & 0.003\end{array}$ & $\begin{array}{l}{[0.960 ; 2.167]} \\
{[0.400 ; 0.834]}\end{array}$ \\
\hline
\end{tabular}

${ }^{*} \mathrm{~B}=\beta,{ }^{*} \mathrm{OR}=$ Odds Ratio, ${ }^{*} \mathrm{CI}=$ Confidence Interval

Table 4 Random-effects model estimation of OR for 24 developing countries.

\begin{tabular}{llllll}
\hline Country Name & Type of Place of Residence & Maternal Education & Father's Education & Living below Poverty Line & Ma \\
\hline \multirow{3}{*}{ Afghanistan } & OR & OR & OR & OR & OR \\
Angola & 0.702 & 0.857 & 0.878 & 0.883 & 1.9 \\
Bangladesh & 0.974 & 0.561 & 0.765 & 0.649 & 1.2 \\
Benin & 0.801 & 0.640 & 0.575 & 0.715 & 1.3 \\
Chad & 1.098 & 0.849 & 0.831 & 0.824 & 1.1 \\
Cambodia & 0.451 & 0.867 & 0.944 & 0.950 & 1.2 \\
Egypt & 0.699 & 0.596 & 0.525 & 0.406 & 0.7 \\
Ethiopia & 0.553 & 0.706 & 0.888 & 0.700 & 0.9 \\
Guinea & 0.544 & 0.759 & 0.790 & 0.692 & 1.1 \\
India & 0.737 & 0.530 & 0.628 & 0.658 & 1.1 \\
& & 0.563 & & 0.588 &
\end{tabular}




\begin{tabular}{llllll}
\hline Country Name & Type of Place of Residence & Maternal Education & Father's Education & Living below Poverty Line & Ma \\
\hline Indonesia & 0.923 & 0.731 & 0.751 & 0.757 & 1.4 \\
Kenya & 1.055 & 0.999 & 0.980 & 1.084 & 1.1 \\
Lesotho & 0.814 & 0.892 & 0.978 & 1.119 & 0.5 \\
Malawi & 0.847 & 0.988 & 0.903 & 0.866 & 1.2 \\
Myanmar & 0.660 & 0.637 & 0.816 & 0.682 & 1.4 \\
Nepal & 0.803 & 0.727 & 0.768 & 0.690 & 0.9 \\
Nigeria & 0.716 & 0.678 & 0.683 & 0.696 & 1.4 \\
Pakistan & 0.765 & 0.644 & 0.755 & 0.729 & 1.1 \\
Sierra Leone & 1.046 & 0.978 & 0.996 & 1.063 & 0.5 \\
South Africa & 1.249 & 0.684 & 2.054 & 0.492 & 1.0 \\
Tanzania & 1.264 & 1.131 & 1.417 & 1.198 & 1.2 \\
Timor-Leste & 0.707 & 0.969 & 0.837 & 0.910 & 0.8 \\
Zambia & 1.114 & 1.119 & 1.007 & 1.029 & 0.730 \\
Zimbabwe & 0.575 & 0.557 & 0.650 & 82.9 & 0.0 \\
$I^{2}$ & 79.3 & 77.2 & 75.9 & 0.041 & 0.0 \\
$\tau^{2}$ & 0.043 & 0.043 & 0.031 & & 8.0 \\
\hline
\end{tabular}

$\hat{\tau}^{2}$ : Estimate of between-study variance

Table 5 Random-effects model estimation (summary effect) for various covariates in 24 developing countries

\begin{tabular}{llll}
\hline Variables & Random-effects model & Random-effects model & Random-effects model \\
\hline & Overall OR & P-Value & $\begin{array}{l}95 \% \text { Confidence Interval } \\
(\mathrm{CI})\end{array}$ \\
$\begin{array}{l}\text { Type of Place of } \\
\begin{array}{l}\text { Residence } \\
\text { Maternal Education }\end{array}\end{array}$ & 0.796 & 0.0001 & {$[0.721 ; 0.877]$} \\
$\begin{array}{l}\text { Father's Education } \\
\text { Living below Poverty }\end{array}$ & 0.762 & 0.0001 & {$[0.690 ; 0.842]$} \\
$\begin{array}{l}\text { Line } \\
\text { Maternal Current }\end{array}$ & 0.784 & 0.0001 & {$[0.750 ; 0.891]$} \\
$\begin{array}{l}\text { Working Status } \\
\text { Birth Order Number }\end{array}$ & 0.909 & 0.0001 & {$[0.715 ; 0.861]$} \\
Maternal Age at & 0.689 & 0.0001 & {$[1.008 ; 1.238]$} \\
$\begin{array}{l}\text { Delivery } \\
\text { Number of Fetus }\end{array}$ & 0.193 & 0.0001 & {$[0.835 ; 0.990]$} \\
Taking ANC & 0.356 & 0.0062 & {$[0.636 ; 0.747]$} \\
$\begin{array}{l}\text { Taking PNC } \\
\text { Size of Child }\end{array}$ & 0.302 & 0.0010 & {$[0.176 ; 0.213]$} \\
\hline
\end{tabular}

The true treatment effect can estimate the average treatment effect that varies from study to study from the random-effects model, illustrated in tables 4 and 5. In this study, we intended to use the random-effects model as the study showed high between-study variations (heterogeneity). About $79.3 \%$ of the variation $\left(\mathrm{I}^{2}\right.$ $=79.3 \%)$ has been observed for the type of place of residence. The overall OR is 0.796 (95\% CI 0.721 to 0.878), which means the individuals residing in urban areas have a $20.4 \%$ lower chance of experiencing infant deaths than their rural counterparts. About $77.2 \%$ of the variation $\left(\mathrm{I}^{2}=77.2 \%\right)$ has been found for maternal education. The overall OR is 0.762 (95\% CI 0.690 to 0.842$)$, meaning mothers who have above primary level education are 0.7622 times or $23.8 \%$ less likely to confront infant death compared to the mothers who have 
up to primary education. Similarly, father's education shows about $75.9 \%$ of the variation with overall OR 0.817 (95\% CI 0.750 to 0.891), which indicates, with an increment in education level, fathers have 0.817 times or $18.3 \%$ lower chance of experiencing infant death. For living below the poverty line, $\mathrm{I}^{2}$ has been found to be $82.9 \%$, where the overall OR is 0.784 (95\% CI 0.715 to 0.861 ), which reveals the odds of infant mortality is 0.784 times or $21.6 \%$ lower to the individuals who don't live below the poverty line compared to those who live below the poverty line. About $82.0 \%$ of the variation $\left(\mathrm{I}^{2}=82.0 \%\right)$ has been observed for maternal current working status. The overall OR is 1.117 (95\% CI 1.008 to 1.238), which means the odds of infant mortality is 1.117 times or $11.7 \%$ higher in the women who are currently working compared to those who are not working currently. For birth order number $\mathrm{I}^{2}$ has been found to be $71.4 \%$ with overall OR 0.909 (95\% CI 0.835 to 0.990 ), indicating a $9.1 \%$ lower chance of infant mortality in the second and above-born child than those who are the firstborn child. The overall OR is 0.689 (95\% CI 0.636 to 0.747 ) for maternal age at delivery with a $47.0 \%$ variation. This suggests that with the increment of a mother's age, the risk of infant death decreases. Again, the overall OR for the number of the fetus is 0.193 (95\% CI 0.176 to 0.213 ) with $53.8 \%$ variation. This indicates that infant death occurs at a rate of 0.193 times or $80.7 \%$ less likely in women carrying a single fetus than those who carry multiple fetuses. About $91.2 \%$ of the variation $\left(\mathrm{I}^{2}\right.$ $=91.2 \%$ ) has been found for taking ANC. The overall OR is 0.356 (95\% CI 0.311 to 0.407 ), which means infant death occurs at 0.356 times or $64.4 \%$ less likely in the women who took antenatal care than those who don't take it. For taking PNC I ${ }^{2}$ has been found to be $92.5 \%$ with overall OR 0.302 (95\% CI 0.243 to 0.375 ), expressing a lower chance of infant mortality in the respondents who are taken for postnatal check-ups. Size of child shows $86.6 \%$ variation $\left(\mathrm{I}^{2}=86.6 \%\right)$ with overall $\mathrm{OR}$ of 0.653 (95\% CI 0.588 to 0.726$)$. This indicates that infant death occurs 0.653 times or $34.7 \%$ less likely in the average-sized child than those who are larger or smaller than average.

Estimation from the meta-analysis in Bangladesh's circumstance displayed in table 4 narrated that urban residents have 0.974 times or $2.6 \%$ of lower chance of confronting infant death compared to the rural residence. Similarly, maternal education, father's education, living below the poverty line, birth order number, and maternal age at delivery are positively associated with infant mortality, with ORs of $0.640,0.575,0.715$, 0,814, and 0.830, respectively. Again, the number of fetus, taking ANC, PNC services, and the child's size is also positively associated with infant mortality, where OR are $0.113,0.314,0.323$, and 0.543 , respectively. We considered the treatment group (category) for the place of residence to be urban, above primary for parent's education level, no for living below the poverty line, yes for maternal working status, $2^{\text {nd }}$ and above born for birth order, above 19 years for maternal age, single for the number of fetus, yes for both of taking ANC, PNC services and the average for the size of the child. However, maternal working status has a noticeable negative influence on infant mortality with an OR of 1.332. Overall, the number of fetus, taking ANC and PNC services are the most significant factors that affect the risk of infant mortality for both methods; random-effects meta-analysis and binary logistic regression for BDHS. 


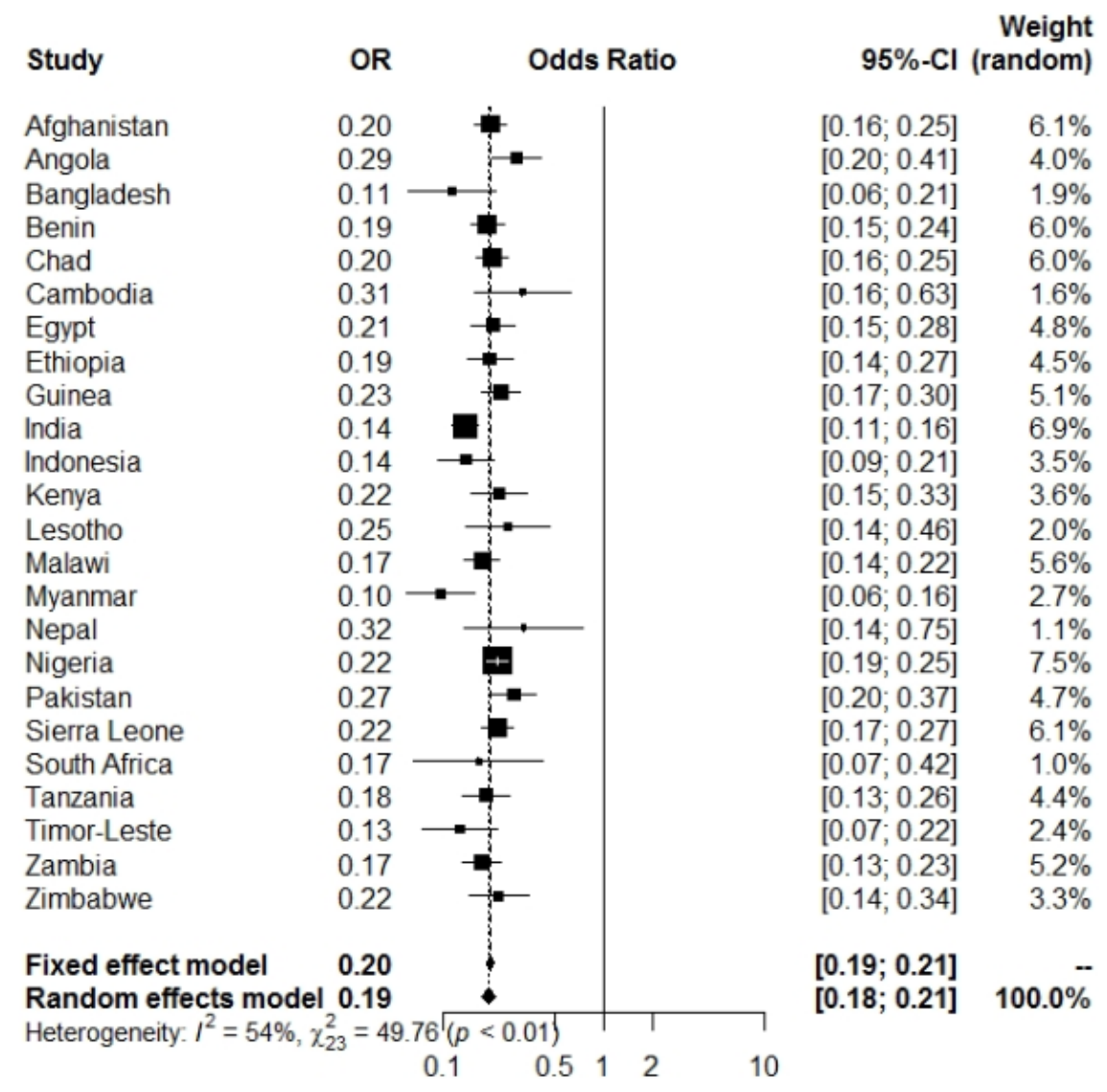

Figure 2 Forest plot for the number of fetus showing the weight of the study by the size of each box while each crossed line indicates $95 \%$ CI.

Figure 2 illustrates that the women of Myanmar carried single fetus have the highest lower chance of confronting infant deaths among all the countries, as the OR is 0.10 with $95 \%$ CI 0.06 to 0.16 . On the other hand, women of Nepal have the lowest lower chance (OR 0.32 with $95 \%$ CI 0.14 to 0.75 ) of experiencing infant mortality with a single fetus. The overall estimate is statistically significant, with a p-value $\leq 0.01$ at a $5 \%$ level of significance.

\section{Discussion}

Through the outcome of the logistic regression model, maternal education, father's education, wealth index, birth order number, maternal age at delivery, number of fetus, taking ANC, PNC, and child's size is found to have a significant influence on infant death for Bangladesh.

In this study, the odds of infant death are lower among births to mothers who have above primary level education than the mothers with up to primary level education for BDHS data. For instance, mothers who had secondary level education are 0.537 times or $46.3 \%$ less likely to confront infant death compared to the mothers who had up to primary education. Finding from the meta-analysis also supports this result. This finding is in line with the outcome of other research ${ }^{23,24,26-29}$. Maternal education may influence child health and mortality with the help of various pathways ${ }^{39}$. This could be, mothers having a secondary and above level of education resulted in better knowledge of health-related services. Besides, better education tends them to live in rich communities with better access to health services ${ }^{26}$.

Both of the logistic regression models for BDHS data and meta-analysis for 24 developing countries revealed 
that as similar to maternal education, the father's higher education reduces the risk of infant mortality. Infant mortality is 0.344 times or $65.6 \%$ less likely to the higher educated fathers than those who have up to primary education (Ref. category) for Bangladesh. From the overall estimate of meta-analysis, it is found that fathers who had above primary level education are 0.8174 times or $18.26 \%$ less likely to experience infant death compared to the fathers who had up to primary education. Some other study supports this finding $26,27,29$. In our social orders, the father is the fundamental pay worker and chief of a family. In this way, the father's education assumes a significant job in earning, which guarantees nutrition, clothing, housing, and so forth. In other words, there might be a direct connection between a father's education and access to youngster wellbeing facilities ${ }^{29}$.

Both the outcome of the logistic regression model for BDHS data and meta-analysis revealed that individuals whose ages are above 19 years could reduce infants' death compared to those who are 19 years or less in Bangladesh, holding the other covariates at a controlled level. From the outcome of BDHS data, we know that the women whose ages were above 19 are 0.477 times or $52.3 \%$ less likely to experience infant mortality than the women whose ages were 19 or less than 19. In a meta-analysis, the overall random effect for maternal age at delivery as treatment expressed about $31 \%$ lower chance of infant death when the individuals are above 19 years old. This result is supported by other findings ${ }^{14,23,24,29}$. Lack of childbearing knowledge might be responsible for the higher risk of infant mortality to the younger mother ${ }^{15}$. Additionally, children born to young mothers were more likely to be premature, have low birth weights, and experiencing complexity at the time of delivery ${ }^{40}$.

The number of fetuses shows a highly significant impact on infant mortality for Bangladesh as the odds ratio is 6.634 for multiple fetuses, which means the risk of infant mortality is 6.634 times higher in cases of women who carried multiple fetuses compared to those who carried a single fetus (Ref. category). Again, from the outcome of the overall meta-analysis estimate, infant death occurs 0.1934 times or $80.66 \%$ less likely in the women who carried a single fetus than those women who carried multiple fetuses. Thus, the odds of infant death were higher among twin births compared with singleton births. This study is supported by a study conducted in Indonesia ${ }^{41}$. The possible explanation for this finding could be pregnant with twin fetuses usually lead to prematurity, which is the most common cause of infant death. Besides, twin to twin transfusion syndrome may occur, which further leads to death ${ }^{4250}$. Also, a twin pregnancy normally ends up with low birth weight, which expands kid weakness to contamination and diminishes their resistance ${ }^{43}$. As a result, child survival is decreased. This investigation was likewise predictable with an examination done by a researcher in Burkina Faso ${ }^{44}$.

Mothers with at least one ANC visit decrease infant death odds compared to mothers with no ANC visits in both cases for Bangladesh and 24 other developing countries. The overall estimate from the meta-analysis uncovered that infant death occurs 0.3560 times or $64.4 \%$ less likely in women who took antenatal care than those who didn't take it. This result is supported by other studies ${ }^{45-47}$. The conceivable explanation could be women having ANC visits get an opportunity of prompt detection of complications and early inception of breastfeeding, which help the invulnerability of a youngster ${ }^{48}$. Likewise, women who had total ANC follow up had expanded the likelihood of conceiving an offspring by the skilled birth attendant, which lessens the chances of infant death ${ }^{49}$. In addition, follow-up with ANC usually leads to having quality essential newborn care, which increases infant survival ${ }^{50}$.

PNC has a higher impact on infant mortality, which indicates the risk of infant mortality is lower in the children who are taken for the postnatal check-ups. The study uncovered that a lower risk of infant death is found in the children who were taken for postnatal care in Bangladesh, holding the other covariates at a controlled level. That is, infant mortality occurs 0.303 times or $69.7 \%$ less likely to the child who was taken for a postnatal check-up than those who did not go for a postnatal check-up. In a meta-analysis, the overall random effect for taking PNC as treatment expressed about a $70 \%$ lower chance of infant death to the children who were taken for postnatal care than those who were not taken for the check-ups. Another finding supports this outcome ${ }^{51}$.

We confronted several limitations while conducting the research. The primary constraint is that we had 
limited access to avail the DHS data because of authorized permission. For this reason, we could only collect data from 24 of the 91 countries from the DHS database. Another limitation is that a bias selection may add to our study as DHS data utilized in this study covered a wider range and different time points. For estimating OR from random-effects meta-analysis, we had to create $2 \times 2$ cross-tabulation for which each variable was categorized into two categories only. Moreover, a wide number of factors that could influence infant mortality could not be included in our study because of the unavailability of those variables in some DHS data.

The study has some strengths despite of these constraints. We combined two methods: binary logistic regression of BDHS data and meta-analysis of 24 DHS data. The integrated findings enlarged the validity of the outcome of the research. We unfolded a new research approach by introducing this special mixed-method design. Because of its extensive and acute quality, better knowledge and insights could be generated.

\section{Conclusion}

Undoubtedly infant mortality as a significant part of under-five child mortality is the most grounded pointer of a nation's prosperity, as it reflects social, monetary, and ecological conditions. Thus, it explicates the standard of life. This study evidently acknowledged the most significant influential factors of infant mortality. The number of fetus, taking ANC and PNC are the dominating factors of infant mortality. Mothers with multiple fetuses should take under proper surveillance. Besides, modernized health care services should be provided to both of rural and urban regions with free medical facilities for unprivileged counterparts. If the required scheme can be implemented, then the destined reduction of infant mortality of the Sustainable Development Goals by 2030 can be met.

\section{References}

1. Blaxter M. The health of the children. A review of research on the place of health in cycles of disadvantage. Heinemann Educational Books.; 1981.

2. Taskaya S, Demirkiran M. Determinants of infant mortality in last two decades: a bibliometric analysis. Int J Community Med Public Health. 2016;3:776-780.

3. Miller NZ, Goldman GS. Infant mortality rates regressed against number of vaccine doses routinely given: Is there a biochemical or synergistic toxicity? Hum Exp Toxicol. 2011;30(9):1420-1428.

4. Vakili R, Emami Moghadam Z, Khademi G, Vakili S, Saeidi M. Child mortality at different world regions: A comparison review. Int $J$ Pediatr. 2015;3(4.2):809-816.

5. Khodaee GH, Khademi G, Saeidi M. Under-five Mortality in the World (1900-2015). Int J Pediatr. 2015;3(6.1):1093-1095.

6. Dadipoor S, Alavi A, Ziapour A, Safari-Moradabadi A. Factors Involved in the Mortality of Infants under the Age of One Year in Bandar Abbas-Iran: A Document-Based Study. Int J Pediatr.2018;6(4):7519-7527.

7. SafariMoradabadi A, Rajaei M, Naderi S. Investigating the underlying causes of mortality among less than one year old infants in pediatric hospital of Bandar Abbas, Iran. Hormozgan Medical Journal.2014;18(3):180186.

8. Kamal SM, Ashrafuzzaman M, Nasreen S. Risk factors of neonatal mortality in Bangladesh. Journal of Nepal Paediatric Society.2012;32(1):37-46.

9. Målqvist M. Neonatal mortality: an invisible and marginalised trauma.Global health action. 2011;4(1):5724.

10. Rajaratnam JK, Marcus JR, Flaxman AD, et al. Neonatal, postneonatal, childhood, and under-5 mortality for 187 countries, 1970-2010: a systematic analysis of progress towards Millennium Development Goal 
4. The Lancet. 2010;375(9730):1988-2008.

11. Titaley CR, Dibley MJ, Agho K, Roberts CL, Hall J. Determinants of neonatal mortality in Indonesia. BMC Public Health.2008;8(1):1-15.

12. Lu Z, Bandara JS, Paramati SR. Impact of sanitation, safe drinking water and health expenditure on infant mortality rate in developing economies. Australian Economic Papers. 2020;59(1):13-33.

13. Organization WH. World health statistics 2019: monitoring health for the SDGs, sustainable development goals. 2019.

14. Karmaker S, Lahiry S, Roy D, Singha B. Determinants of infant and child mortality in Bangladesh: time trends and comparisons across South Asia. Bangladesh Journal of Medical Science. 2014;13(4):431-437.

15. Schell CO, Reilly M, Rosling H, Peterson S, Mia Ekström A. Socioeconomic determinants of infant mortality: a worldwide study of 152 low-, middle-, and high-income countries. Scandinavian journal of public health. 2007;35(3):288-297.

16. bank W. Countries and Economies. 2019; https://data.worldbank.org/country.

17. Hug L, Dharrow D, Zhong K, You D. Levels and trends in child mortality: Report 2018. The World Bank;2018.

18. Sartorius BK, Sartorius K. Global infant mortality trends and attributable determinants-an ecological study using data from 192 countries for the period 1990-2011. Population Health Metrics.2014;12(1):1-15.

19. Rubayet S, Shahidullah M, Hossain A, et al. Newborn survival in Bangladesh: a decade of change and future implications. Health Policy Plan. 2012;27(suppl_3):iii40-iii56.

20. Group WB. World development report 2016: digital dividends.World Bank Publications; 2016.

21. National Institute of Population Research and Training, Mitra and Associates, and ICF International. Bangladesh Demographic and Health Survey 2014: Key Indicators. Dhaka, Bangladesh, and Rockville, Maryland, USA: NIPORT, Mitra and Associates, and ICF International;2015.

22. Lamichhane R, Zhao Y, Paudel S, Adewuyi EO. Factors associated with infant mortality in Nepal: a comparative analysis of Nepal demographic and health surveys (NDHS) 2006 and 2011. BMC Public Health.2017;17(1):1-18.

23. Huda TM, Tahsina T, Arifeen SE, Dibley MJ. The importance of intersectoral factors in promoting equity-oriented universal health coverage: a multilevel analysis of social determinants affecting neonatal infant and under-five mortality in Bangladesh. Global health action. 2016;9(1):29741.

24. Ezeh OK, Agho KE, Dibley MJ, Hall JJ, Page AN. Risk factors for postneonatal, infant, child and under-5 mortality in Nigeria: a pooled cross-sectional analysis. BMJ open. 2015;5(3):e006779.

25. Ntenda PAM, Chuang K-Y, Tiruneh FN, Chuang Y-C. Factors associated with infant mortality in Malawi. Journal of Experimental \& Clinical Medicine. 2014;6(4):125-131.

26. Abuqamar M, Coomans D, Louckx F. The impact of parental education on infant mortality in Gaza strip, Palestine. Journal of Public Health and Epidemiology. 2011;3(1):28-33.

27. Chowdhury QH, Islam R, Hossain K. Socio-economic determinants of neonatal, post neonatal, infant and child mortality. International Journal of Sociology and Anthropology. 2010;2(6):118-125.

28. Kumar PP, File G. Infant and child mortality in Ethiopia: a statistical analysis approach. Ethiopian Journal of Education and Sciences. 2010;5(2).

29. Mondal MNI, Hossain MK, Ali MK. Factors influencing infant and child mortality: A case study of Rajshahi District, Bangladesh. Journal of Human Ecology. 2009;26(1):31-39. 
30. Program TD. https://dhsprogram.com/data/data-collection.cfm, 2020.

31. Tranmer M, Elliot M. Binary logistic regression. Cathie Marsh for census and survey research, paper. $2008 ; 20$.

32. Cramer JS. The origins of logistic regression. 2002.

33. Rücker G, Schwarzer G, Carpenter JR, Schumacher M. Undue reliance on I 2 in assessing heterogeneity may mislead. BMC Med Res Methodol.2008;8(1):1-9.

34. Higgins JP, Thompson SG, Deeks JJ, Altman DG. Measuring inconsistency in meta-analyses. BMJ. 2003;327(7414):557-560.

35. DerSimonian R, Laird N. Meta-analysis in clinical trials. Control Clin Trials. 1986;7(3):177-188.

36. Borenstein M, Hedges LV, Higgins JP, Rothstein HR. A basic introduction to fixed-effect and randomeffects models for meta-analysis. Research synthesis methods. 2010;1(2):97-111.

37. Verhagen A, Ferreira ML. Forest plots. J Physiother.2014;60(3):170-173.

38. Szumilas M. Explaining odds ratios. Journal of the Canadian academy of child and adolescent psychiatry. 2010;19(3):227.

39. Hobcraft J. Women's education, child welfare and child survival: a review of the evidence. Health Transit Rev. 1993:159-175.

40. Pandey A. Infant and child mortality in India. 1998.

41. Febriyuna N. Determinants of Infant Mortality in Indonesia. The Hague, The Netherlands. 2015.

42. Hehir MP, Mctiernan A, Martin A, Carroll S, Gleeson R, Malone FD. Improved perinatal mortality in twins-changing practice and technologies. Am J Perinatol. 2016;33(1):84-89.

43. Townsend R, Khalil A. Fetal growth restriction in twins. Best Practice $\mathcal{E} 3$ Research Clinical Obstetrics E Gynaecology. 2018;49:79-88.

44. Becher H, Muller O, Jahn A, Gbangou A, Kynast-Wolf G, Kouyate B. Risk factors of infant and child mortality in rural Burkina Faso.Bull World Health Organ. 2004;82:265-273.

45. Hollowell J, Oakley L, Kurinczuk JJ, Brocklehurst P, Gray R. The effectiveness of antenatal care programmes to reduce infant mortality and preterm birth in socially disadvantaged and vulnerable women in high-income countries: a systematic review. BMC Pregnancy Childbirth. 2011;11(1):1-20.

46. McDermott J, Steketee R, Larsen S, Wirima J. Syphilis-associated perinatal and infant mortality in rural Malawi. Bull World Health Organ. 1993;71(6):773.

47. Partridge S, Balayla J, Holcroft CA, Abenhaim HA. Inadequate prenatal care utilization and risks of infant mortality and poor birth outcome: a retrospective analysis of 28,729,765 US deliveries over 8 years. Am J Perinatol. 2012;29(10):787.

48. Phukan D, Ranjan M, Dwivedi L. Impact of timing of breastfeeding initiation on neonatal mortality in India. International breastfeeding journal. 2018;13(1):1-10.

49. Rarani MA, Rashidian A, Khosravi A, Arab M, Abbasian E, Morasae EK. Changes in socio-economic inequality in neonatal mortality in Iran between 1995-2000 and 2005-2010: an Oaxaca decomposition analysis.International journal of health policy and management.2017;6(4):219.

50. Tafere TE, Afework MF, Yalew AW. Does antenatal care service quality influence essential newborn care (ENC) practices? In Bahir Dar City Administration, North West Ethiopia: a prospective follow up study.Ital J Pediatr. 2018;44(1):1-8. 
51. Baqui AH, Ahmed S, El Arifeen S, et al. Effect of timing of first postnatal care home visit on neonatal mortality in Bangladesh: a observational cohort study. BMJ. 2009;339.

Table 1 Baseline characteristics of different variables with levels for BDHS data

\begin{tabular}{|c|c|c|c|}
\hline Variable & $\begin{array}{l}\text { Levels of the variables } \\
\text { with code }\end{array}$ & Frequency & Percentage \\
\hline $\begin{array}{l}\text { Dependent variable } \\
\text { Infant mortality }\end{array}$ & No [0] Yes [1] & 4561163 & 96.53 .5 \\
\hline $\begin{array}{l}\text { Covariates Type of } \\
\text { place of residence }\end{array}$ & Rural [0] Urban [1] & 32201504 & 68.231 .8 \\
\hline Maternal education & $\begin{array}{l}\text { Up to primary }[0] \\
\text { Secondary }[1] \text { Higher }[2]\end{array}$ & 19592224541 & 41.547 .011 .5 \\
\hline Father's education & $\begin{array}{l}\text { Up to primary }[0] \\
\text { Secondary }[1] \text { Higher }[2]\end{array}$ & 25261478720 & 53.531 .315 .2 \\
\hline Wealth index & $\begin{array}{l}\text { Poorest [0] Poorer [1] } \\
\text { Middle [2] Richer [3] } \\
\text { Richest [4] }\end{array}$ & 1011902901980930 & 21.419 .119 .120 .719 .7 \\
\hline $\begin{array}{l}\text { Maternal current working } \\
\text { status }\end{array}$ & No [0] Yes [1] & 36891035 & 78.121 .9 \\
\hline Birth order number & $\begin{array}{l}\text { First born [0] Second and } \\
\text { above [1] }\end{array}$ & 19432781 & 41.158 .9 \\
\hline Maternal age at delivery & $<=19[0]$ Above $19[1]$ & 11683556 & 24.775 .3 \\
\hline Number of fetus & Single [0] Multiple [1] & 466262 & 98.71 .3 \\
\hline Taking ANC & $\begin{array}{l}\text { No [0] } 1-4 \text { visits [1] More } \\
\text { than } 4 \text { visits }[2]\end{array}$ & 12042573947 & 25.554 .520 .0 \\
\hline Taking PNC & No [0] Yes [1] & 18162908 & 38.461 .6 \\
\hline Size of child & $\begin{array}{l}\text { Average [0] Larger than } \\
\text { average [1] Smaller than } \\
\text { average [2] }\end{array}$ & 3181616927 & 67.413 .019 .6 \\
\hline
\end{tabular}

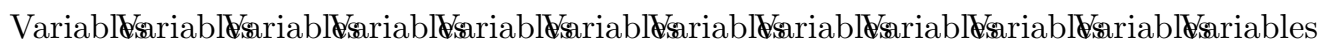

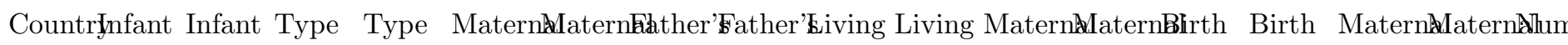

Name mor- mor- of of Ed- Ed- Ed- Ed- be- be- Cur- Cur- Or- Or- Age Age of $\begin{array}{llllllllllll}\text { tal- } & \text { tal- } & \text { Place } & \text { Place } & \text { uca- } & \text { uca- uca- } & \text { uca- low low rent rent der der at } & \text { at } & \text { Fe- } \\ \text { ity } & \text { ity } & \text { of } & \text { of } & \text { tion tion tion tion } & \text { PovertyPovertyWork- Work- Num- Num- De- } & \text { De- } & \text { tus }\end{array}$ $\begin{array}{llllllllllll}\mathrm{n} & \mathrm{n} & \text { Res- } & \text { Res- } & \mathrm{n} & \mathrm{n} & \mathrm{n} & \mathrm{n} & \text { Line } & \text { Line ing ing ber ber liv- liv- } & \mathrm{n}\end{array}$

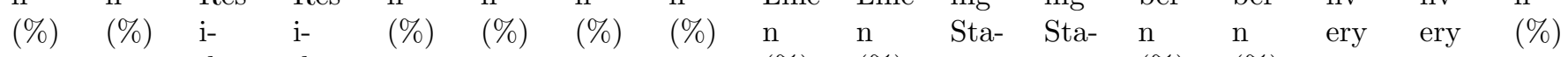
$\begin{array}{llllllllll}\text { dence dence } & (\%) & (\%) & \text { tus } & \text { tus } & (\%) & (\%) & \mathrm{n} & \mathrm{n}\end{array}$

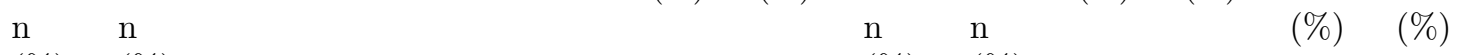
(\%) (\%) $\quad(\%) \quad(\%)$

No Yes Rural Urban Up Above Up Above No Yes Not Working ${ }^{\text {st }} 2^{\text {nd }} \quad[?] 19$ Above Sing to primarto primary working born \& years 19 primary primary above

$\begin{array}{llllllllllllllllll}\text { Sierra } & 9453 & 778 & 7424 & 2807 & 8879 & 1352 & 7659 & 2572 & 5590 & 4641 & 2416 & 7815 & 1720 & 8511 & 1150 & 9081 & 9777\end{array}$ Leone (92.4) (7.6) (72.6) (27.4) (86.8) (13.2) (74.9) (25.1) (54.6) (45.4) (23.6) 976.4) (16.8) (83.2) (11.2) (88.8) (95. 2013 
$\begin{array}{lllllllllllllllll}\text { Zambial1164 } & 469 & 7425 & 4208 & 8061 & 3572 & 5748 & 5885 & 5997 & 5636 & 4761 & 6872 & 1880 & 9753 & 1233 & 10400 & 1123\end{array}$

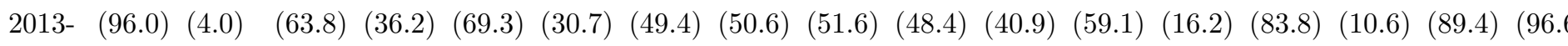
14

$\begin{array}{lllllllllllllllll}\text { Banglad5661 } & 163 & 3220 & 1504 & 1959 & 2765 & 2526 & 2198 & 2811 & 1913 & 3689 & 1035 & 1943 & 2781 & 1168 & 3556 & 4662\end{array}$

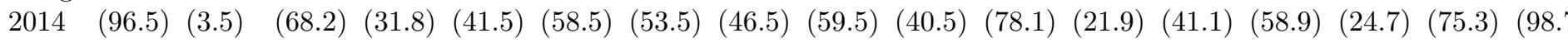
$\begin{array}{lllllllllllllllll}\text { Cambo69.3 } & 164 & 5148 & 1929 & 4504 & 2573 & 3732 & 3345 & 4072 & 3005 & 2360 & 4717 & 2744 & 4333 & 491 & 6586 & 6945\end{array}$ $\begin{array}{lllllllllllllllllll} & 014 & (97.7) & (2.3) & (72.7) & (27.3) & (63.6) & (36.4) & (52.7) & (47.3) & (57.5) & (42.5) & (33.3) & (66.7) & (38.8) & (61.2) & (6.9) & (93.1) & (98 .\end{array}$

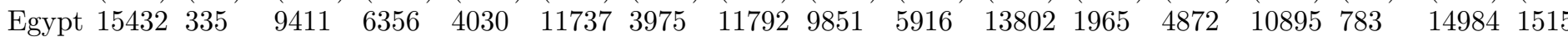
$\begin{array}{llllllllllllllllll} & & & \end{array}$ $\begin{array}{lllllllllllllllll}\text { Kenya } 8886 & 311 & 6169 & 3028 & 6978 & 2219 & 6141 & 3056 & 4140 & 5057 & 3794 & 5403 & 1777 & 7420 & 780 & 8417 & 8960\end{array}$ $\begin{array}{lllllllllllllllllll} & 2014 & (96.6) & (3.4) & (67.1) & (32.9) & (75.9) & (24.1) & (66.8) & (33.2) & (45.0) & (55.0) & (41.3) & (58.7) & (19.3) & (80.7) & (8.5) & (91.5) & (97 .\end{array}$ $\begin{array}{lllllllllllllllll}\text { Lesoth } 8565 & 164 & 2071 & 658 & 1408 & 1321 & 1784 & 945 & 1426 & 1303 & 1913 & 816 & 953 & 1776 & 314 & 2415 & 2656\end{array}$

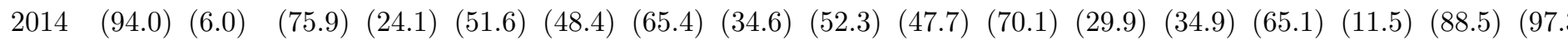

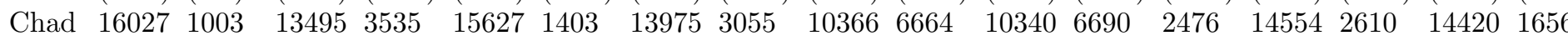

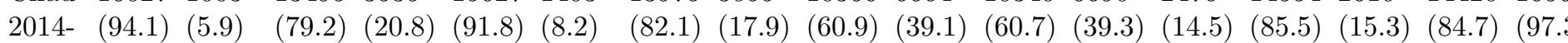
15 $2015 \quad(95.8)(4.2)$ Zimbab49d 4213 $2015 \quad(95.8)(4.2)$ Angola8788 423 2015- (95.4) (4.6) 16

Malawi13603 522 2015- (96.3) (3.7) 16 16 16 16 2016 2016 (96.9) (3.1) Timor-5580 154 Leste (97.3) (2.7) 2016

Indonesi6487 347 2017 (97.9) (2.1) Benin 11379549 2017- (95.4) (4.6) 18

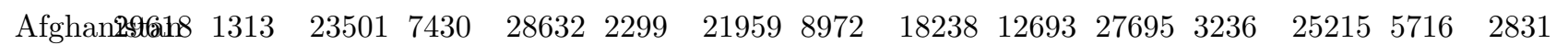

$\begin{array}{lllllllllllllll}\text { Tanzan8a91 } & 319 & 6668 & 1842 & 7033 & 1477 & 6798 & 1712 & 4707 & 3803 & 1962 & 6548 & 1733 & 6777 & 843\end{array}$ 2015- (96.3) (3.7) (78.4) (21.6) (82.6) (17.4) (79.9) (20.1)

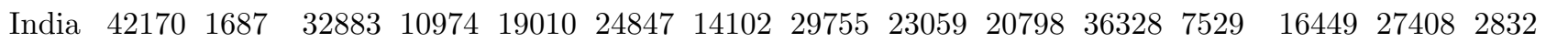
2015- (96.2) (3.8) (75.0) (25.0) (43.3) (56.7) (32.2) (67.8) (52.6) (47.4) (82.8) (17.2) (37.5) (62.5) (6.5)

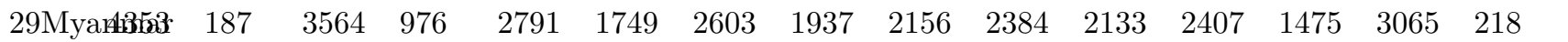

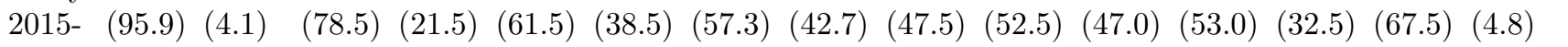

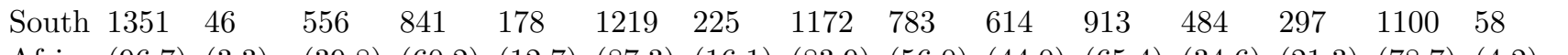

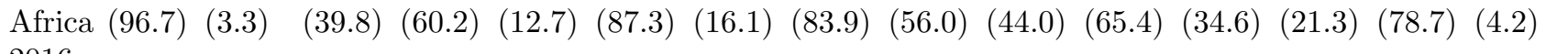

$\begin{array}{lllllllllllllll}\text { Ethiopi2372 } & 455 & 8099 & 1728 & 8811 & 1016 & 8053 & 1774 & 4464 & 5363 & 7246 & 2581 & 1874 & 7953 & 866\end{array}$ $\begin{array}{lllllllllllllllll}2 & 016 & (95.4) & (4.6) & (82.4) & (17.6) & (89.7) & (10.3) & (81.9) & (18.1) & (45.4) & (54.6) & (73.7) & (26.3) & (19.1) & (80.9) & (8.8)\end{array}$ $\begin{array}{llllllllllllllll}\text { Nepal } & 4821 & 155 & 2145 & 2831 & 2642 & 2334 & 1815 & 3161 & 2596 & 2380 & 2399 & 2577 & 1958 & 3018 & 760\end{array}$

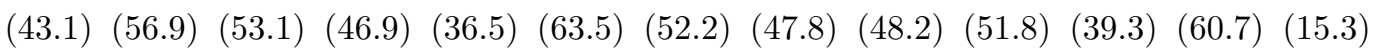
$\begin{array}{lllllllllllll}3801 & 1933 & 2229 & 3505 & 2290 & 3444 & 3697 & 2037 & 3604 & 2130 & 1453 & 4281 & 275\end{array}$

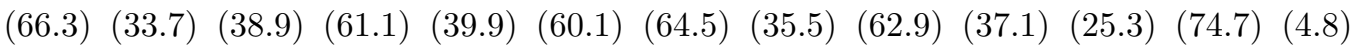

$84528382 \quad 4330$ $\begin{array}{lllllllllllll}(50.2) & (49.8) & (25.7) & (74.3) & (27.2) & (72.8) & (53.8) & (46.2) & (53.8) & (46.2) & (32.5) & (67.5) & (4.8)\end{array}$

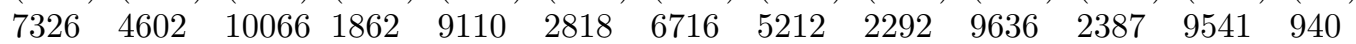

281003036 (90.8) $(98$. 46184940 (90.1) $(96$. $\begin{array}{lllllllllllllll}4081 & 5130 & 6666 & 2545 & 4532 & 4679 & 4594 & 4617 & 2489 & 6722 & 1488 & 7723 & 1214 & 7997 & 8939 \\ (44.3) & (55.7) & (72.4) & (27.6) & (49.2) & (50.8) & (49.9) & (50.1) & (27.0) & (73.0) & (16.2) & (83.8) & (13.2) & (86.8) & (97 .(\end{array}$ $\begin{array}{lllllllllllllll}11892 & 2233 & 11176 & 2949 & 9132 & 4993 & 8115 & 6010 & 4878 & 9247 & 3330 & 10795 & 1891 & 12234 & 1359\end{array}$ (84.2) (15.8) (79.1) (20.9) (64.7) (35.3) (57.5) (42.5) (34.5) (65.5) (23.6) (76.4) (13.4) (86.6) (2) $667 \quad 8211$ (90.1) $(96$. 410254309 (93.5) $(98$. $1322 \quad 4455$ 95.2) $(98$. 3391358 89619563 (91.2) $(97$. $4216 \quad 4909$ (84.7) $(98$. $5459 \quad 562$ (95.2) (98. 160311659 (95.2) (98. 109881134 (92.1) $(95$. 


\begin{tabular}{|c|c|c|c|c|c|c|c|c|c|c|c|c|c|c|c|}
\hline $\begin{array}{l}\text { Pakistan1845 } \\
2017-\quad(94.9) \\
18\end{array}$ & $\begin{array}{l}638 \\
(5.1)\end{array}$ & $\begin{array}{l}6960 \\
(55.8)\end{array}$ & $\begin{array}{l}5523 \\
(44.2)\end{array}$ & $\begin{array}{l}8086 \\
(64.8)\end{array}$ & $\begin{array}{l}4397 \\
(35.2)\end{array}$ & $\begin{array}{l}5204 \\
(41.7)\end{array}$ & $\begin{array}{l}7279 \\
(58.3)\end{array}$ & $\begin{array}{l}6785 \\
(54.4)\end{array}$ & $\begin{array}{l}5698 \\
(45.6)\end{array}$ & $\begin{array}{l}11046 \\
(88.5)\end{array}$ & $\begin{array}{l}1437 \\
(11.5)\end{array}$ & $\begin{array}{l}2964 \\
(23.7)\end{array}$ & $\begin{array}{l}9519 \\
(76.3)\end{array}$ & $\begin{array}{l}786 \\
(6.3)\end{array}$ & $\begin{array}{l}11697 \\
(93.7)\end{array}$ \\
\hline Guinea6854 & 462 & & & 6518 & 798 & & & & & & & & & & \\
\hline (93.7) & $(6.3)$ & & $(26.5)$ & (89.1) & (10.9) & & & & & & & & & & \\
\hline ria29359 & 950 & 0664 & 10645 & 19339 & 11970 & 15963 & 15346 & 16608 & 14701 & 10278 & 21031 & & 25684 & & 28387 \\
\hline (93.8) & $(6.2)$ & (66.0) & $(34.0)$ & (61.8) & $(38.2)$ & $(51.0)$ & $(49.0)$ & $(53.0)$ & $(47.0)$ & (32.8) & $(67.2)$ & $(18.0)$ & $(82.0)$ & $(9.3)$ & (90.7) \\
\hline
\end{tabular}

Table 2 Baseline characteristics of selected covariates for 24 developing countries

Table 3 Results of the binary logistic regression model affecting socio-economic and demographic factors for infant mortality in Bangladesh.

\begin{tabular}{|c|c|c|c|c|}
\hline & $B^{*}$ & Odds Ratio & $p$-value & $95 \%$ CI for OR \\
\hline $\begin{array}{l}\text { Type of place of } \\
\text { residence } \\
\text { Rural(Ref. } \\
\text { Category) Urban }\end{array}$ & -0.101 & 0.904 & 0.606 & {$[0.616 ; 1.326]$} \\
\hline $\begin{array}{l}\text { Maternal } \\
\text { Education Up to } \\
\text { Primary (Ref. } \\
\text { Category) } \\
\text { Secondary Higher }\end{array}$ & $-0.622-0.723$ & 0.5370 .485 & $0.0020 .000 \quad 0.116$ & $\begin{array}{l}{[0.380 ; 0.759]} \\
{[0.197 ; 1.196]}\end{array}$ \\
\hline $\begin{array}{l}\text { Father's } \\
\text { Education Up to } \\
\text { Primary (Ref. } \\
\text { Category) } \\
\text { Secondary Higher }\end{array}$ & $-0.413-1.067$ & 0.6620 .344 & 0.0170 .0400 .014 & $\begin{array}{l}{[0.447 ; 0.980]} \\
{[0.147 ; 0.807]}\end{array}$ \\
\hline $\begin{array}{l}\text { Wealth Index } \\
\text { Poorest (Ref. } \\
\text { Category) Poorer } \\
\text { Middle Richer } \\
\text { Richest }\end{array}$ & $\begin{array}{ll}-0.802 & -0.897 \\
-0.209 & -0.049\end{array}$ & $\begin{array}{l}0.4480 .4080 .812 \\
0.952\end{array}$ & $\begin{array}{l}0.000 \quad 0.000 \quad 0.000 \\
0.3890 .874\end{array}$ & $\begin{array}{l}{[0.303 ; 0.663]} \\
{[0.254 ; 0.654]} \\
{[0.505 ; 1.305]} \\
{[0.521 ; 1.741]}\end{array}$ \\
\hline $\begin{array}{l}\text { Maternal } \\
\text { Current Working } \\
\text { Status Not } \\
\text { Working (Ref. } \\
\text { Category) Working }\end{array}$ & 0.027 & 1.027 & 0.883 & {$[0.716 ; 1.475]$} \\
\hline $\begin{array}{l}\text { Birth Order } \\
\text { Number First } \\
\text { Born (Ref. } \\
\text { Category) Second } \\
\text { and above }\end{array}$ & -1.016 & 0.362 & 0.000 & {$[0.248,0.527]$} \\
\hline $\begin{array}{l}\text { Maternal Age at } \\
\text { Delivery } \\
<=19 \text { (Ref. } \\
\text { Category) Above } 19\end{array}$ & -0.741 & 0.477 & 0.000 & {$[0.333 ; 0.682]$} \\
\hline $\begin{array}{l}\text { Number of Fetus } \\
\text { Single (Ref. } \\
\text { Category) Multiple }\end{array}$ & 1.892 & 6.634 & 0.000 & {$[3.247 ; 13.555]$} \\
\hline
\end{tabular}




\begin{tabular}{lllll}
\hline & $B^{*}$ & Odds Ratio & $p$-value & $95 \%$ CI for OR \\
\hline Taking & $-1.306-0.758$ & 0.2710 .468 & 0.0000 .0000 .003 & {$[0.192 ; 0.382]$} \\
Antenatal Care & & & & {$[0.286 ; 0.768]$} \\
No (Ref. & & & \\
Category) 1-4 & & & & \\
$\begin{array}{l}\text { Visits More than } \\
4 \text { Visits }\end{array}$ & & 0.303 & & \\
$\begin{array}{l}\text { Taking Postnatal } \\
\text { Care No (Ref. }\end{array}$ & -1.194 & & & \\
$\begin{array}{l}\text { Category) Yes } \\
\text { Size of Child }\end{array}$ & $0.366-0.548$ & 1.4430 .578 & 0.0010 .0780 .003 & {$[0.960 ; 2.167]$} \\
$\begin{array}{l}\text { Average (Ref. } \\
\text { Category) Larger }\end{array}$ & & & & {$[0.400 ; 0.834]$} \\
than Average & & & \\
$\begin{array}{l}\text { Smaller than } \\
\text { Average }\end{array}$ & & & & \\
\hline
\end{tabular}

${ }^{*} \mathrm{~B}=\beta,{ }^{*} \mathrm{OR}=\mathrm{Odds}$ Ratio, ${ }^{*} \mathrm{CI}=$ Confidence Interval

Table 4 Random-effects model estimation of OR for 24 developing countries.

\begin{tabular}{|c|c|c|c|c|}
\hline Country Name & Type of Place of Residence & Maternal Education & Father's Education & Living below Poverty Line \\
\hline & OR & OR & OR & OR \\
\hline Afghanistan & 0.608 & 0.857 & 0.878 & 0.883 \\
\hline Angola & 0.702 & 0.561 & 0.765 & 0.649 \\
\hline Bangladesh & 0.974 & 0.640 & 0.575 & 0.715 \\
\hline Benin & 0.801 & 0.849 & 0.831 & 0.824 \\
\hline Chad & 1.098 & 0.867 & 0.944 & 0.950 \\
\hline Cambodia & 0.451 & 0.596 & 0.525 & 0.406 \\
\hline Egypt & 0.699 & 0.706 & 0.888 & 0.700 \\
\hline Ethiopia & 0.553 & 0.759 & 0.790 & 0.692 \\
\hline Guinea & 0.544 & 0.530 & 0.661 & 0.658 \\
\hline India & 0.737 & 0.563 & 0.628 & 0.588 \\
\hline Indonesia & 0.923 & 0.731 & 0.751 & 0.757 \\
\hline Kenya & 1.055 & 0.999 & 0.980 & 1.084 \\
\hline Lesotho & 0.814 & 0.892 & 0.978 & 1.119 \\
\hline Malawi & 0.847 & 0.988 & 0.903 & 0.866 \\
\hline Myanmar & 0.660 & 0.637 & 0.816 & 0.682 \\
\hline Nepal & 0.803 & 0.727 & 0.768 & 0.690 \\
\hline Nigeria & 0.716 & 0.678 & 0.683 & 0.696 \\
\hline Pakistan & 0.765 & 0.644 & 0.755 & 0.729 \\
\hline Sierra Leone & 1.046 & 0.978 & 0.996 & 1.063 \\
\hline South Africa & 1.249 & 0.684 & 2.054 & 0.492 \\
\hline Tanzania & 1.264 & 1.131 & 1.417 & 1.198 \\
\hline Timor-Leste & 0.707 & 0.969 & 0.837 & 0.910 \\
\hline Zambia & 1.114 & 1.119 & 1.007 & 1.029 \\
\hline Zimbabwe & 0.575 & 0.557 & 0.650 & 0.730 \\
\hline$I^{2}$ & 79.3 & 77.2 & 75.9 & 82.9 \\
\hline$\tau^{2}$ & 0.043 & 0.043 & 0.031 & 0.041 \\
\hline
\end{tabular}


$\hat{\tau}^{\mathbf{2}}$ : Estimate of between-study variance

Table 5 Random-effects model estimation (summary effect) for various covariates in 24 developing countries

\begin{tabular}{llll}
\hline Variables & Random-effects model & Random-effects model & Random-effects model \\
\hline & Overall OR & P-Value & $\begin{array}{l}95 \% \text { Confidence Interval } \\
(\mathrm{CI})\end{array}$ \\
$\begin{array}{l}\text { Type of Place of } \\
\begin{array}{l}\text { Residence } \\
\text { Maternal Education }\end{array}\end{array}$ & 0.796 & 0.0001 & {$[0.721 ; 0.877]$} \\
$\begin{array}{l}\text { Father's Education } \\
\text { Living below Poverty }\end{array}$ & 0.762 & 0.0001 & {$[0.690 ; 0.842]$} \\
Line & 0.817 & 0.0001 & {$[0.750 ; 0.891]$} \\
Maternal Current & 1.117 & 0.0001 & {$[0.715 ; 0.861]$} \\
$\begin{array}{l}\text { Working Status } \\
\text { Birth Order Number }\end{array}$ & 0.909 & 0.0001 & {$[1.008 ; 1.238]$} \\
Maternal Age at & 0.689 & 0.0001 & {$[0.835 ; 0.990]$} \\
$\begin{array}{l}\text { Delivery } \\
\text { Number of Fetus }\end{array}$ & 0.193 & 0.0062 & {$[0.636 ; 0.747]$} \\
Taking ANC & 0.356 & 0.0010 & {$[0.176 ; 0.213]$} \\
$\begin{array}{l}\text { Taking PNC } \\
\text { Size of Child }\end{array}$ & 0.302 & 0.0001 & {$[0.311 ; 0.407]$} \\
\hline
\end{tabular}

Figures 


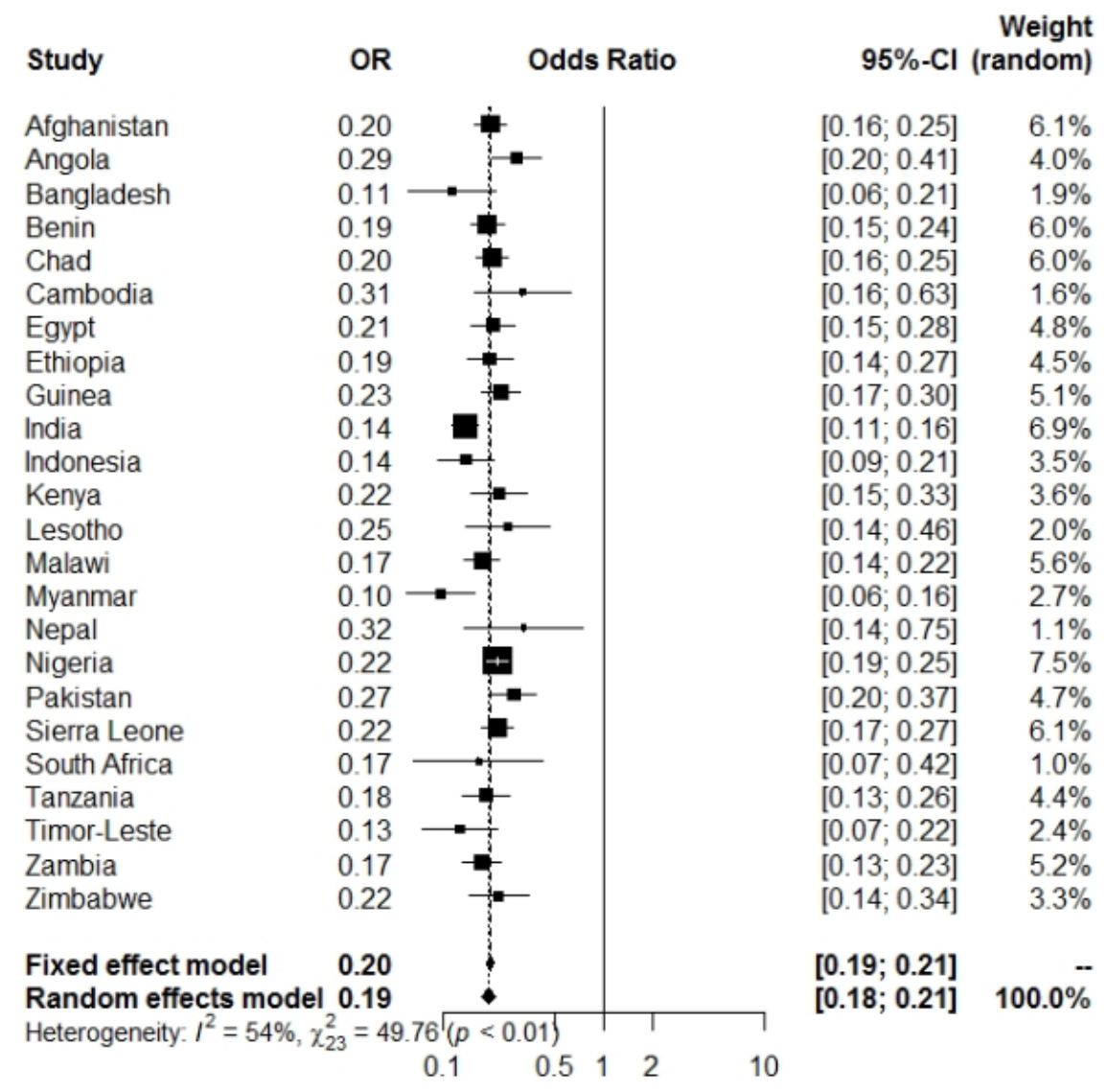

Figure 2 Forest plot for the number of fetus showing the weight of the study by the size of each box while each crossed line indicates $95 \%$ CI. 